slowing down and raising the level of the voice.

\section{Articulation}

Figure 1 shows the mean number of errors by all Ss per 100 words of the 1,500-word primary message and clearly shows the markedly larger number of errors made under Condition $\mathrm{C}$ as compared to Condition B. A one-tail $t$ test shows the poorer articulation after Word 1,000 to be significant, $p<.05$ $(t=1.914, \mathrm{df}=11)$. The rise in errors was mainly an increase in the number of omissions rather than within-word errors.

Figure 2 shows that the rise in the mean number of errors was not due simply to a few highly susceptible Ss. The data from all Ss have been split up into two equal groups on the basis of the number of errors made under Condition D (DAF reading). Those who made the most errors constituted the high-susceptibility group, and, conversely, those who were less affected by DAF made up the low-susceptibility group. Although the differences in error scores for the two groups is considerable, the inability to completely ignore DAF-even for Ss of low-susceptibility-is shown.

\section{Latency}

The ear-voice lag did not increase significantly when the irrelevant message was changed to DAF. The mean latency over 1,000 words of simple dichotic shadowing was about 1.5 words, compared with Treisman \& Geffen's (1967) figure of 3.1 words. This difference might have occurred because the present experiment involved intrinsic practice by virtue of the long passages or because Treisman's Ss had additional attentional loads.

\section{Correlation of Variables}

There were strong correlations between the three error rates for each $S$ under Condition $B$ (simple dichotic shadowing), Condition C (DAF shadowing), and Condition D (DAF reading). Ear-voice latency during DAF shadowing was correlated with the error rate (rho $=0.69$, $\mathrm{p}<.02$ ).

\section{CONCLUSIONS}

It appears impossible for Ss to erect a "Broadbent filter" which would enable them to ignore the speech-disruptive effects of DAF. Carey (1969) has suggested that filtering theories and, in particular, filter attenuation are untenable in the light of such data, but Treisman's model would allow for this result. Treisman (1961) proposed that nonattended channels are "attenuated" ("that is, made less easily discriminable") but are nonetheless facilitated at the dictionary stage of the model if they are "probable, "important," or recently received." DAF would be covered by this last category because the $\mathrm{S}$ monitors the direct auditory feedback, and it is this processing that the DAF confuses, causing the speech disruption. It has yet to be shown that direct auditory feedback is essential for speaking; these results are consistent with the view that all speech production tasks require some attention to the immediate feedback and with the view that each individual has a speech production-perception system of which the feedback system is relevant to a normal speaking rate.

\section{REFERENCES}

CAREY, P. W. Delayed auditory feedback and the shadowing response. Unpublished $\mathrm{PhD}$ dissertation. Harvard University, 1969.

CHERRY, E. C. Some experiments on the recognition of speech with one and with two ears. Journal of the Acoustical Society of America, 1953, 25, 975-979.

FAIRBANKS, G. Selective vocal effects of delayed auditory feedback. Journal of Speech \& Hearing Disorders, 1955, 20, 333-346.

MORAY, N. Effect of relative intensities of messages in dichotic shadowing. Language \& Speech, 1958, 1, 110-113.

TREISMAN, A. M. Attention and speech. Unpublished PhD dissertation. Oxford University, 1961.

TREISMAN, A. M., \& GEFFEN, G. Selective attention: Perception or response? Quarterly Joumal of Experimental Psychology, 1967, 19, 1-16.

YATES, A. J. Delayed auditory feedback. Psychological Bulletin, 1963, 60, 213-251.

YATES, A. J. Delayed auditory feedback and shadowing. Quarterly Journal of Experimental Psychology, 1965, 17, 125-131.

\title{
Reinforcement of verbal behavior by evaluative meaning words
}

\author{
CLAIRE F. ETAUGH, RICHARD J. KELLIHER, and RICHARD B. STALLING \\ Bradley University, Peoria, Ill. 61606
}

This study tested the generality of an earlier finding that evaluative meaning words function as reinforcers for children's instrumental motor learning. College students served as $\mathrm{Ss}$ in a verbal conditioning task. Words rated as having positive, negative, or neutral evaluative meaning (EM) were presented contingent upon the use of self-referent pronouns and were later removed during extinction. Frequency of emission of self-referent pronouns: (1) increased, following presentation of positive EM words and removal of negative EM words; (2) decreased, following removal of positive, and presentation of negative, EM words; (3) was unaffected by presentation or removal of neutral EM words. The results strengthen Staats's (1968) conclusion that EM words can function as reinforcers and punishers in instrumental tasks.

Staats (1968) has suggested that the evaluative portion of a word's meaning (Osgood, Suci, \& Tannenbaum, 1957) is established through classical conditioning, and that words which have acquired evaluative meaning will function in instrumental conditioning as reinforcers and punishers. Using sixth-grade children as Ss, Finley \& Staats (1967) demonstrated the reinforcing function of evaluative meaning (EM) words in a button-pushing task; response-contingent positive EM words strengthened the motor response, negative EM words decreased it, and neutral words had no effect on response frequency.

It follows from Staats's position that evaluative meaning words should serve as reinforcers for a wide variety of instrumental behaviors and $S$ populations. The generality of this hypothesis was examined in the present study in a verbal conditioning task with adult Ss.

In addition, while Finley \& Staats (1967) demonstrated that presentation of EM words affects acquisition of an instrumental behavior, the effect of terminating such presentations has not been explored. The present study sought to determine if termination of EM word presentations would produce response extinction, as does the removal of more conventional reinforcers.

\section{SUBJECTS}

Forty-five male undergraduates at Bradley University were assigned randomly 


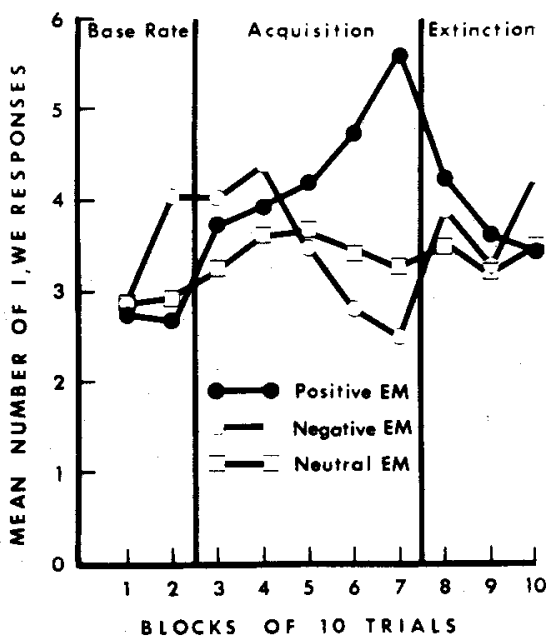

Fig. 1. Mean number of $I$ and We responses as a function of trials for positive, negative, and neutral evaluative meaning (EM) groups.

to one of three EM conditions: positive, negative, or neutral. An additional group of 40 male students from the same population rated the evaluative meaning of the words used in the experiment proper.

\section{SELECTION OF EM WORDS}

Two hundred and one common words were presented in list form to the rating group. As in Finley and Staats's study, each word was rated along a 7-point scale ranging from "very pleasant" (1) to "very unpleasant" (7). Thirty words, 10 in each EM category, which were among those used by Finley and Staats, were selected for use in the present experiment. The positive words (mean rating $=2.31$ ) were cheerful, famous, fun, holiday, brave, laughter, joy, dollar, happiness, and angel; the negative words (mean rating $=5.54$ ) were guilty, ugly, pain, sad, hate, thief, shock, hurt, worry, and poison; and the neutral words (mean rating $=3.86$ ) were trunk, every, stem, cover, bridge, bone. brick, those, section, and moment.

\section{TASK}

One hundred common past-tense verbs were selected. The verbs previously had been rated by another sample of Bradley University undergraduates as connoting a neutral impression (Etaugh, 1969). Each verb was typed on an index card above a list of six pronouns (I, We, He, She, You, and They) whose respective orders were randomized for the series of 100 cards.

\section{PROCEDURE}

Each $S$ was told that he was participating in a sentence-formation study. The $\mathrm{S}$ was first shown a sample card and instructed to make up a sentence containing the indicated verb and beginning with any one of the pronouns.
The $S$ constructed sentences until the series of 100 cards was completed. Ten cards constituted one block of 10 trials.

In order to establish a base rate of response, no EM words were presented following S's responses during the first two blocks of trials. During Blocks 3-7 (acquisition), an EM word appropriate to S's condition was spoken by $E$ in a flat unemotional tone at the end of any sentence which S began with I or We. The particular EM word used by $E$ was determined by a prearranged random order which differed for each $S$. During Blocks 8-10 (extinction), EM words were no longer delivered, regardless of S's response.

\section{RESULTS}

The mean number of $I$ and We responses emitted on each block of trials by each EM group is shown in Fig. 1. The data were evaluated by means of an analysis of variance based on the number of $I$ and $W e$ responses emitted on Blocks 1 and 2 (base-rate trials), Block 7 (final acquisition trials), and Block 10 (final extinction trials). The only significant $F$ ratio was that for the EM Conditions by Trials interaction $(F=14.99, \mathrm{df}=4,84: \mathrm{p}<.01)$. Further analysis of this interaction was carried out by means of Scheffe's (1953) tests ( $p<.05$ for all comparisons). First, performance of Ss in the three conditions was compared on each block of trials. No differences were found in the number of $I$ and $W e$ responses emitted either during the base-rate blocks or on the final extinction block. On the last acquisition block, however, the positive EM group showed reliably more conditioning than did the neutral EM group, which in turn emitted reliably more $I$ and $W e$ responses than did the negative EM group. Second, performance means of Ss in each condition were compared over blocks of trials. As expected, the positive EM group emitted reliably more I and We responses at the end of acquisition than during the base-rate period, and returned to base level of responding during extinction. Performance of the negative EM group predictably was depressed significantly during acquisition, returning to and actually surpassing base level of responding during extinction. The neutral EM group showed no reliable change in performance throughout the experiment, as expected.

\section{DISCUSSION}

The acquisition data of the present study are in agreement with those of Finley \& Staats (1967) in demonstrating both the reinforcing and punishing properties of words of differing evaluative meaning. During the acquisition period, positive EM words increased the frequency of the responses they followed. negative EM words decreased the frequency of the responses they followed. and neutral words had no effect on response frequency. This replication of Finley and Staats's results. obtained with a different task and $\mathrm{S}$ population. increases the generality of Staats's (1968) conclusion that evaluative meaning words can function as reinforcers and punishers in instrumental tasks.

This conclusion is further supported by the present extinction data. Removal of response-contingent positive EM words produced the same decline in response strength obtained in other verbal conditioning studies (e.g., Greenspoon. 1955) when more conventionally used verbal reinforcers (e.g. mm-hmm, good) are withdrawn. Similarly, removal of response-contingent negative EM words was followed by an increase in the strength of a previously depressed response. much like the removal of a mildly punishing stimulus. Removal of neutral words had no effect.

It should be noted that most of the words used in the present study possess denotative (i.e., referential) meaning, as well as connotative (in this case, evaluative) meaning (Osgood et al, 1957). Previous studies of the effect of verbal reinforcers on the modification of verbal behavior (see review by Kanfer. 1968) typically have used reinforcers (mm-hmm, good) which have an almost purely evaluative meaning. The present findings suggest that, in verbal exchanges between individuals, it is not only the limited category of purely evaluative words which shapes the content of conversations but, in addition, any response-contingent denotative words which also possess an evaluative component.

\section{REFERENCES}

ETALGH, C. F. Experimenter and subject variables in verbal conditioning. Psychological Reports, 1969, 25, 575-580.

FINLEY, J. R., \& STAATS, A. W. Evaluative meaning of words as reinforcement stimuli. Journal of Verbal Learning \& Verbal Behavior, 1967, 6, 193-197.

GREENSPOON. J. The reinforcing effect of two spoken sounds on the frequency of two responses. American Joumal of Psychology, $1955,68,409-416$

KANFER, F. H. Verbal conditioning: A review of its current status. In T. R. Dixon and D. L. Horton (Eds.) Verbal behavior and general behavior theory. Englewood Cliffs, New Jersey: Prentice-Hall, 1968. Pp. 254-290.

OSGOOD, C. E., SUCI, G. J., \& TANNENBALM, $\mathrm{P}$. $\mathrm{H}$. The measurement of meaning. Urbana, Illinois: University of Illinois Press, 1957.

SCHEFFE, H. A method for judging all contrasts in the analysis of variance. Biometrika, 1953, 40, 87-104. 